\title{
Efficiency of different control strategies in a force-feedback gripper
}

\author{
O. B. Lőrinczi, T. Szalay \& P. Aradi \\ Faculty of Mechanical Engineering, \\ Budapest University of Technology and Economics, Hungary
}

\begin{abstract}
This paper aims to introduce the development of a control system implemented for a force-feedback gripper mechanism, as well as the results of the experiments carried out on the device. The achievements shall be used in additional research related to medical purpose grippers, such as artificial hands. The aim of the current research is the improvement of the control system of artificial hands in order to assure the expected usability in the case of a simplified mechanical structure, and eventually to decrease the manufacturing cost of such devices to gain higher availability. First, the main units of the experimental device will be described. After the clarification of the basics, the applied control strategies shall be discussed in detail. From the basic PI/PID control, to adaptive fuzzy/neurofuzzy control, different methods are applied also on the simulation model and the experimental device itself. The main results of the research are the summary of differences between the particular control strategies. The properties of the controls, like speed, stability, accuracy and adaptivity are determined and a comparative analysis have been expounded. From the achieved results, the overall efficiency of the different control strategies can be determined, the type and properties of the optimal control can be described. Finally the aspects of further development will be discussed; on one hand, that means the design and production of a new, more complex mechanism; and on the other hand, the feedback of more state variables in order to gain more information for better function. Hopefully a complete five-finger gripper will be manufactured in the near future, and by the time it will be available, the results of this study can be utilized for its control system.
\end{abstract}

Keywords: biomechatronics, sensor technologies, force-feedback grasping. 


\section{Introduction}

In almost every mechatronics-related task the properties of the control system represent the main field of interest. Since there are many types of control methods, it is important to determine and use the most suitable algorithm for the specific purpose. In this case, the goal is to analyse the behaviour of a force controlled gripper mechanism while different types of algorithms are being applied to control the system.

In everyday practice, the traditional PI and PID controls are widely spread, but there is a range of tasks where these methods are insufficient. That is always caused by rapidly changing boundary conditions and significant nonlinearities in the controlled plant.

For the aforementioned complex systems, advanced control strategies are being developed in order to achieve effective and proper operation. One important feature of these advanced algorithms is the ability of adaptation to various conditions. Fuzzy type controllers are widely spread in the field of adaptive controls [1, 2].

The field of use is wide, because the same control strategy can be applied in industrial tasks, but also in medical applications or other topics. With the progression and spreading of telemanipulation, and its special medical applications like telesurgery [3], the demand for reliable control systems had arisen, and researches are present. Besides telesurgery, the use of haptic information is also a studied area, for example rehabilitation robotics [4, 5].

All the above mentioned fields relate - and thus lead to - the topic of artificial hands [6]. In this subject, the position and force feedbacks are equally important, and the changing conditions necessitate the application of adaptive control algorithms.

The research presented in this paper combines the achievements and possibilities of the aforementioned areas. The goal is the development of an artificial hand that use artificial muscle type actuators and is controlled by an adaptive fuzzy or neuro-fuzzy algorithm. The gripper mechanism that is the subject of this study is a first experiment to this goal.

The target of the performed experiments was to prove that the improvement of the control software result in high upswing with regards to usability and control properties.

Formerly, a two-finger gripper mechanism had been developed in order to investigate the possibilities of force-feedback grasping. For this mechanism, the means of grip force measurement and the control software had to be implemented.

The main problem with the former, traditional proportional integrating control system was the high sensitivity to the rigidity of the gripped material. The controller had to be re-tuned for different type of materials to avoid unstable operation. This is inappropriate for devices operating under continuously changing conditions.

During the research, the PI controller proved to be insufficient in many cases, which justify the need of developing an advanced control system for the 


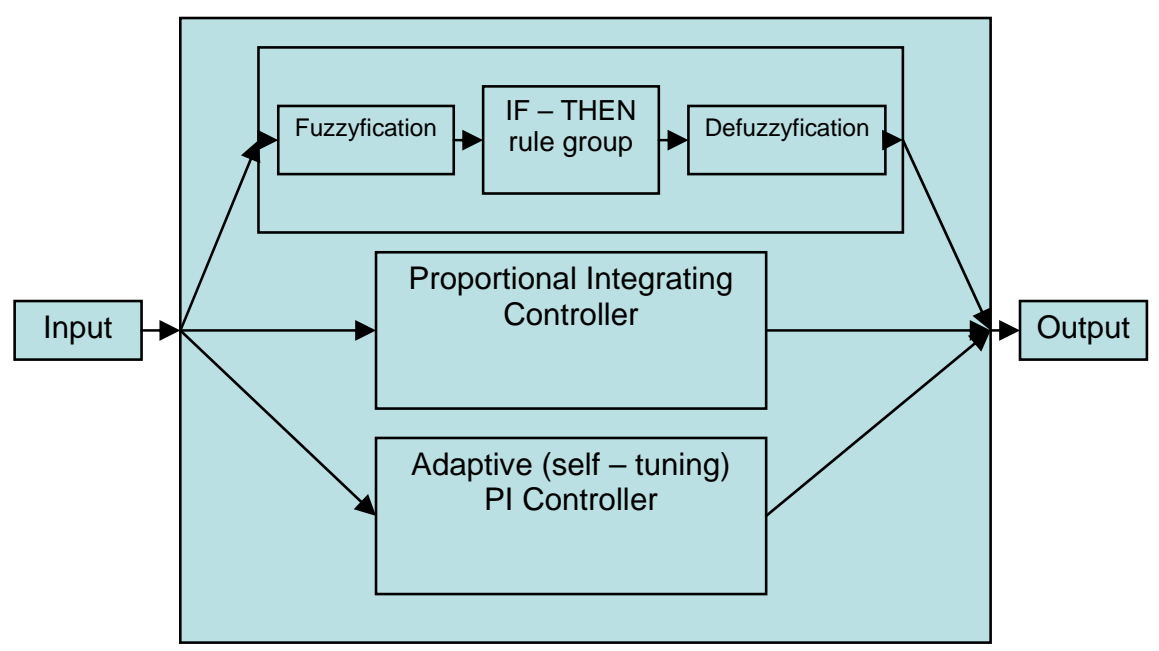

Figure 1: $\quad$ The experiment plan

experimental device. Presently, the implementation of a fuzzy control seems to be the viable option.

\section{The experimental setup}

Most hardware of the system have been developed and produced at the Budapest University of Technology and Economics. The experiments had been carried out using the following devices:

Experiment hardware

- $\quad$ Two-fingered gripper mechanism equipped with strain gauges

- Instrumental amplifier for the measurement

- $\quad$ Power electronics for the DC motor drive

- $\quad$ I/O unit for measurement data acquisition and control

Experiment software

- Control algorithm, running on PC

Figure 2 shows the architecture of the complete system. The arrows indicate the direction of data and energy stream. The instrumental amplifier and the power electronics are placed on the same printed circuit board in order to optimize the structure. The I/O unit used for control and data acquisition is a National Instruments USB-6008. 


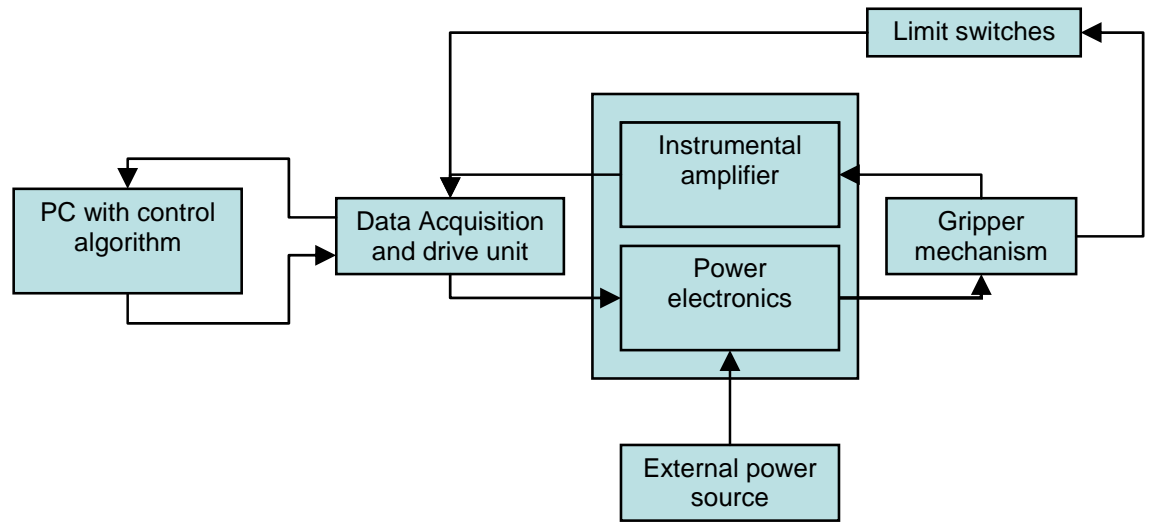

Figure 2: $\quad$ Scheme of the experimental setup

The strain gauges had to be calibrated to achieve precise measurement. The calibration had been carried out by using a weight set. During the measurement, the linear characteristics of the strain gauge had been verified; all measurement points coincided with the linear regression. The measurement had been carried out between $0.5-10 \mathrm{~N}$.

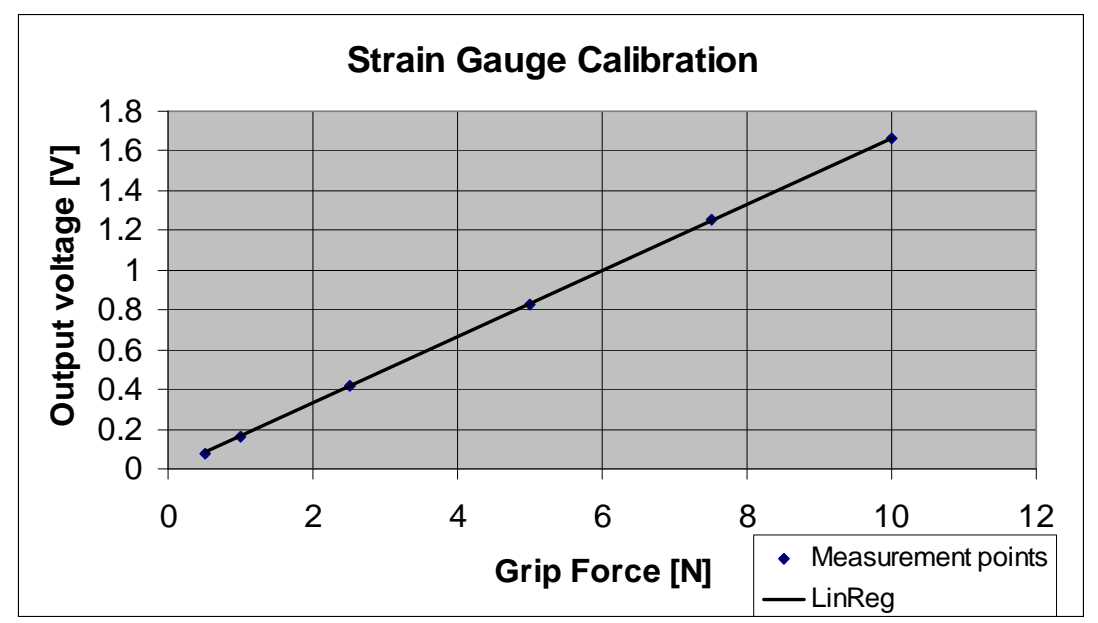

Figure 3: Calibration of the strain gauge.

During calibration, the axis of gravity was parallel to the axis of measurement, thus the systematic error from the dead weight of the gauge had to be compensated. Systematic errors in a measurement can be eliminated completely, so the accuracy of the calibration accords to the accuracy of the AD converter of the data acquisition unit. 


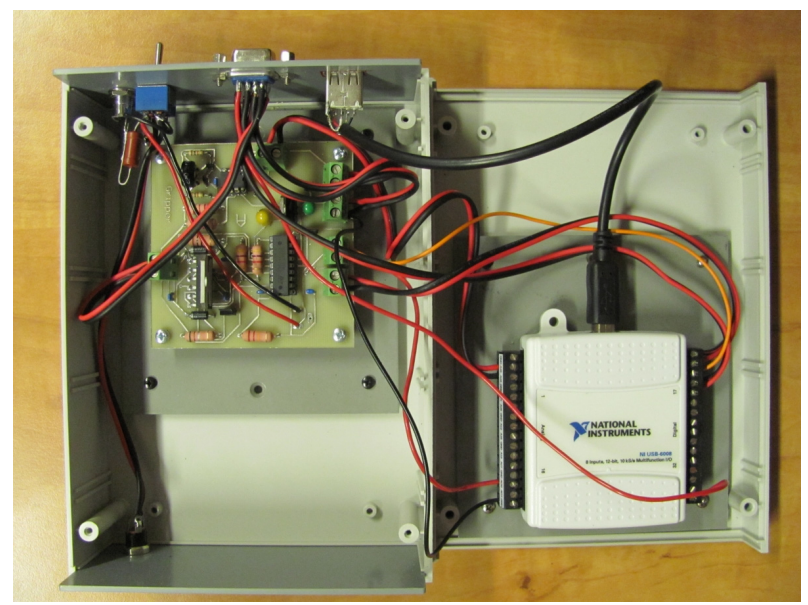

Figure 4: $\quad$ Electronics of the experimental device

The final task before the measurement was the compensation of the offset error of the instrumental amplifier. Since this is also a systematic and static error, it can be compensated by a constant value integrated into the control software.

\section{Control software and measurement methods}

The algorithms controlling the gripper are implemented in LabVIEW and the TUBSim simulation toolkit [7]. Although there are built-in controllers in different toolboxes, the traditional proportional-integrating (PI), the adaptive PI, and the fuzzy controllers are self-developed.

The measurement can be implemented the same way in the case of any controller, also the safety features and actuation are identical for all cases.

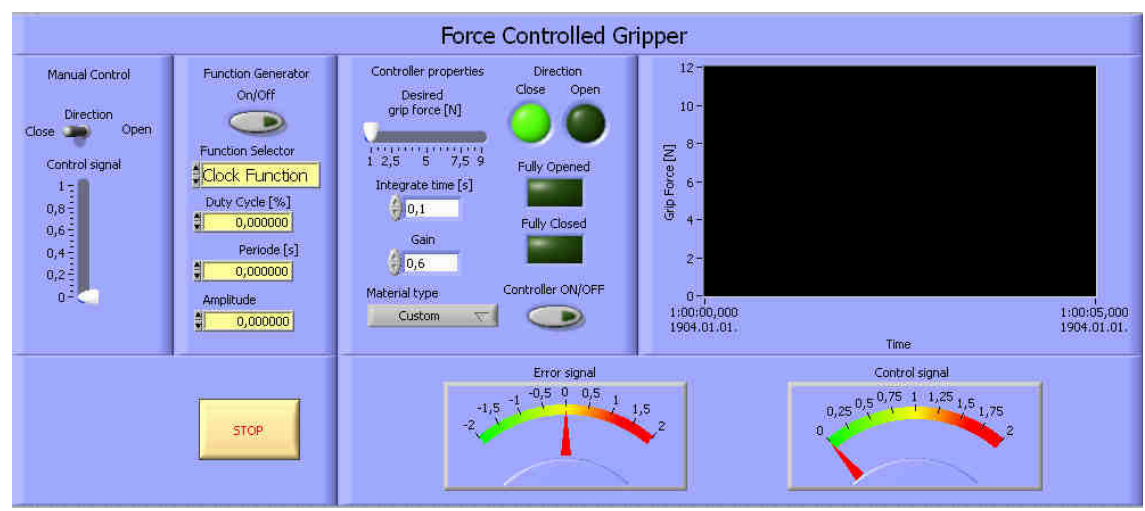

Figure 5: $\quad$ User interface of the control software 
The control algorithms have been examined in three different aspects; control speed, accuracy, stability. Each experiment has been repeated with two different objects to be held; an elastic and a rigid one. As it can be seen among the experiment results, the main difference between the algorithms appears in the case of a change between the flexible and rigid objects.

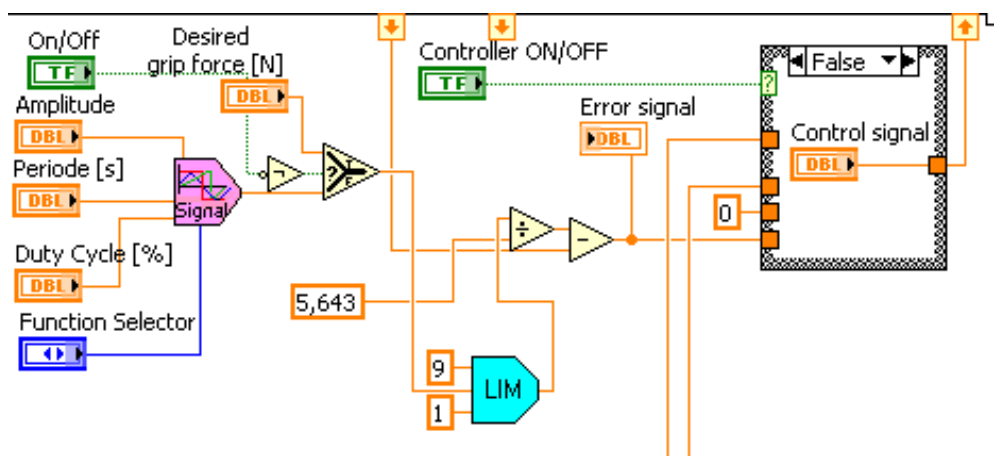

Figure 6: Detail of the control software

\section{Experiment results}

\subsection{Settling time}

In stable operation, the settling time of the control was not highly dependent on the type of the controller. Settling time varied between $0,3-0,8$ sec while less than $5 \%$ overshoot occurred.

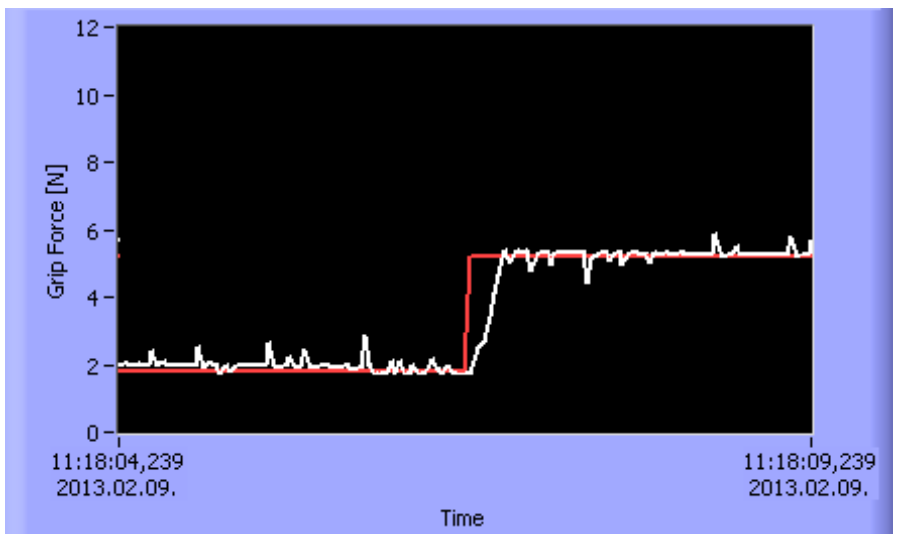

Figure 7: $\quad$ Snapshot of the control in operation

It can be stated, that the settling time correlated more with the tuning of the controller rather than its type. In this aspect, the performance of all controller types (PI, adaptive PI and fuzzy) was equally good. 


\subsection{Control accuracy}

The steady state error of the control loop was zero in all cases. The necessity of that can be proved by using the description of system and controller types. The steady state error depends on the system type, the controller type and the applied input signal. In this case, the input is a step signal and the system is a type 1 system. Assuming these conditions, the value of the steady state error can only be zero [8]. Due to this, the difference between the steady state accuracy of the controllers would only appear under such conditions that are not present during normal operation.

\subsection{Control stability}

The stability of the control was the aspect, where the main anomaly appeared between the different controllers. The simple PI controller could not handle any changes in the flexibility of the gripped object; the properties had to be set to comply with the actual criteria.

A stability loss had appeared when the flexible object had been replaced with the rigid object. In this case, only the flexibility of the levers remained in the system. The adaptive controllers had been able to tune the control properties in a few seconds to maintain the control stability.

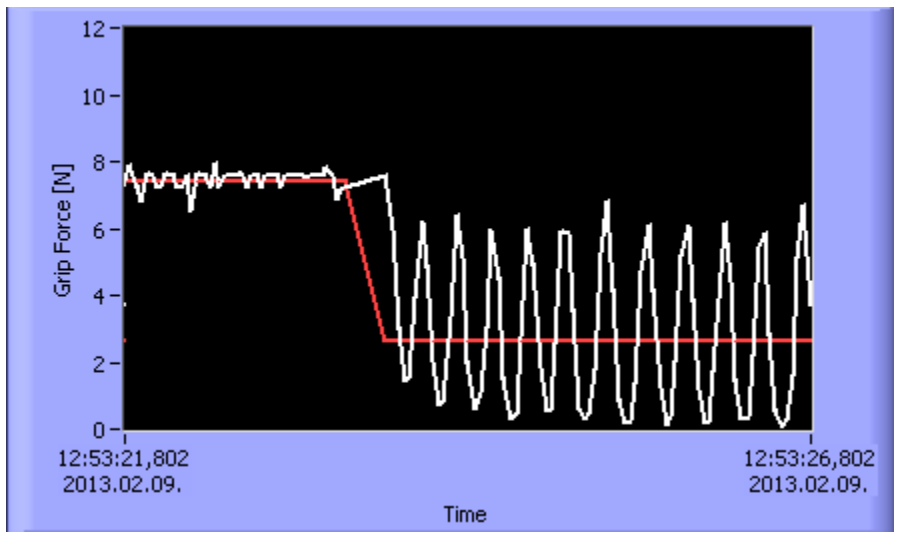

Figure 8: $\quad$ Loss of stability during the experiment.

However the stability can be recovered, a smoother transition is desirable in order to ensure the grip continuity. The filtering of the signal is also necessary to avoid incorrect action due to salient values caused by measurement noise.

In summary, the advantages of advanced control algorithms mainly appear in the stability of the system. The fuzzy controller has more potential, although the feedback of further signals - such as the velocity of the gripper levers or the slip detection of the gripped object - has to be implemented in order to exploit all additional possibilities. 


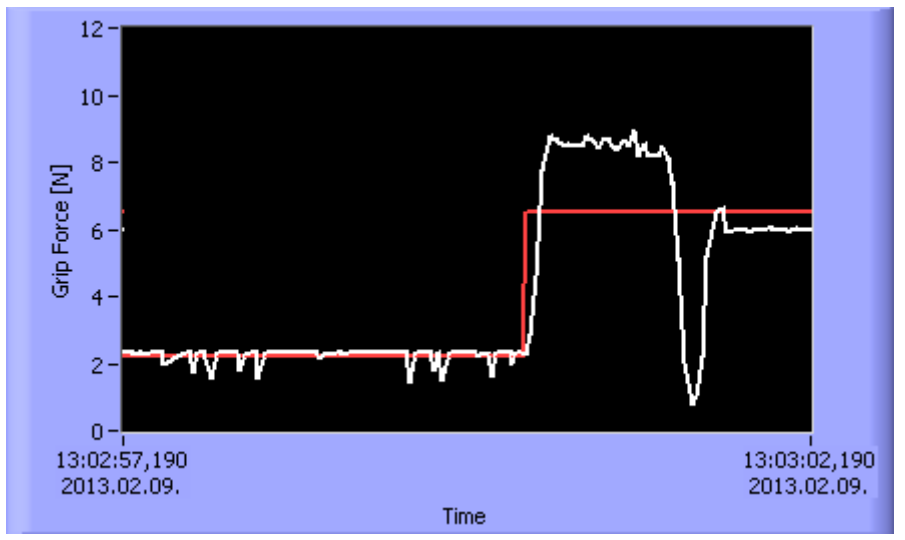

Figure 9: Recovering the control stability.

\section{Future development}

For further improvement, additional variables have to be monitored such as the rotational speed of the DC motor and the position of the levers. The detection of slip between the levers and the held object is also a way of advancement. With such developments, the usability shall be extended and the system can be highly improved.

Refinement of the experiment mechanics is also necessary, especially with regards to the wobbling of the worm gear. This behaviour results in serious nonlinearity, which is usually the most difficult problem also from the aspect of the mathematical model, and the actual control algorithm.

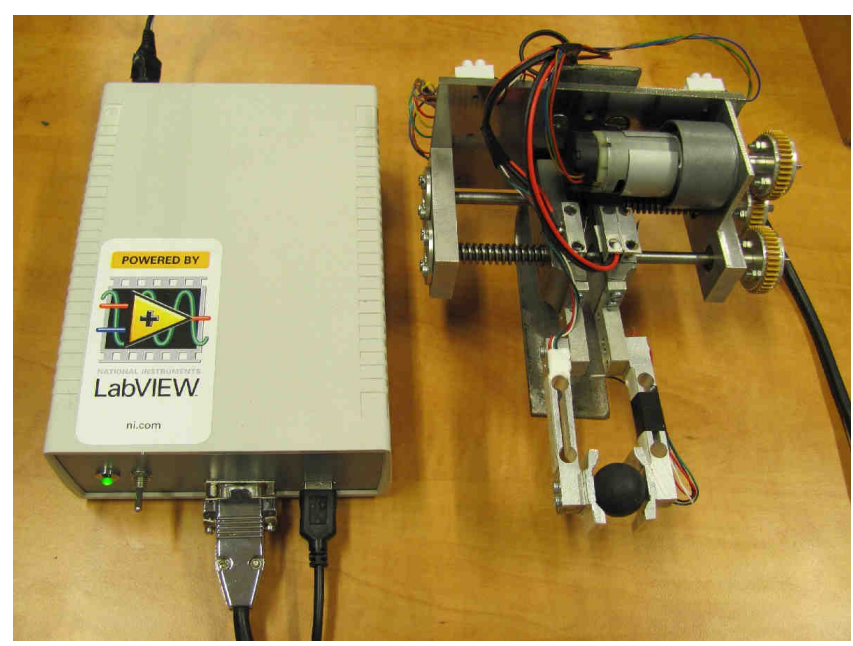

Figure 10: $\quad$ The experimental device during measurement. 


\section{Conclusion}

In this paper, a force feedback gripper, and its control system had been discussed. First the experimental setup, then the types of control algorithms had been introduced.

Based on the measurements, the properties of the control strategies are registered and evaluated. It is clear that the advanced, adaptive algorithms can result in improved performance, but it can be stated that the measurement of further properties is inevitable for large-scale progress.

The means of force measurement and control can be applied in related researches. The results achieved in the framework of this project shall be used in the intelligent corset programme [9].

\section{Acknowledgement}

The authors would like to thank the National Development Agency (NDA) of the Hungarian Government for their support since this study has been carried out commonly as part of the project GERINCO2 TECH_08-A1/2-2008-0121.

\section{References}

[1] Nuno Mendes, Pedro Neto, J. Norberto Pires, A. Paulo Moreira: Fuzzy-PI Force Control for Industrial Robotics, Trends in Intelligent Robotics, Communications in Comp. and Inf. Science, Vol103, 2010, pp. 322-329.

[2] Bing Wei Gao, Jun Peng Shao, Gui Hua Han, Gui Tao Sun, Xiao Dong Yang, Di Wu: Using Fuzzy Switching to Achieve the Smooth Switching of Force and Position, Appl. Mechanics and Materials (Vol. 274), pp. 638-641.

[3] Thomas Hansen, Claus T. Henningsen, Jens J. M. Nielsen, Rasmus Pedersen, John Schwensen, Senthuran Sivabalan, Jesper A. Larsen, John Leth: Implementing force-feedback in a telesurgery environment using parameter estimation, IEEE International Conference on Control Applications (CCA), 2012, pp. 859-864.

[4] Y. Kobayashi, P. Moreira, C. Liu, P. Poignet, N. Zemiti, M. G. Fujie: Haptic feedback control in medical robots through fractional viscoelastic tissue model, Engineering in Medicine and Biology Society, EMBC, 2011 Annual International Conference of the IEEE, pp. 6704-6708.

[5] A. M. M. Ali, R. Ambar, M. M. A. Jamil, A. J. M. Wahi, S. Salim: Artificial hand gripper controller via Smart Glove for rehabilitation process, International Conference on Biomedical Engineering (ICoBE), 2012, pp. 300-304.

[6] Shinji Matsubara, Shingo Okamoto, Jae Hoon Lee: Prosthetic Hand Using Shape Memory Alloy Type Artificial Muscle, Proceedings of the International MultiConference of Engineers and Computer Scientists, IMECS 2012 
[7] Lipovszki G, Aradi P: Simulating Complex Systems and Processes in LabVIEW. Journal Of Mathematical Sciences (New York) 132:(5) 2006, pp. 629-636.

[8] Okko H. Bosgra, Huibert Kwakernaak, Gjerrit Meinsma: Design Methods for Control Systems, Dutch Institute of Systems and Control, 2001

[9] Petra Aradi, Norbert Szakály, József Molnár, Péter Tamás: Intelligent Corset. Submitted to BIOMED 2013, Budapest, April 26-28, 2013. 